\title{
Digital health needs assessment at a national-subnational level in Sri Lanka
}

\author{
Y.A.K.E. Samaranayake ${ }^{1}$, N.P. Liyanarachchi ${ }^{1}$, C. Weerabaddana ${ }^{1}$, K.K.P. Sylva ${ }^{1}$, M.G.P.S.De Silva ${ }^{1}$, R.A.D.L.M. \\ Ranwala $^{1}$, M.S. Manoj ${ }^{1}$, M.U.T. Perera ${ }^{1}$, W.M.A. Wijekoon ${ }^{2}$, P.V.A.I. Gunawardena ${ }^{3}{ }^{*}$ A.U. Amarathunga ${ }^{3}$, \\ C.M. Manawadu ${ }^{3}$, S.D. Siriwardane ${ }^{3}$
}

1 Ministry of Health Nutrition \& Indigenous Medicine, Sri Lanka 2 Provincial Health Information Unit, North Western Province, Sri Lanka 3 Postgraduate Institute of Medicine, Colombo, Sri Lanka

*arsenic1st@hotmail.com

https://orcid.org/0000-0002-2971-2648

\begin{abstract}
Introduction: Healthcare stakeholders hold diverse opinions and needs. Different Inputs, opinions and concerns of the stakeholders are essential to build a sustainable and successful digital health strategy. Successful digital initiatives are based on the understanding of the user characteristics, needs and challenges. Collaborating with stakeholders, sharing information and insights, sharing strategies and resources across projects/organizations leads to increased efficiency and impact. The aim was to assess the national to subnational level digital health needs of the government health sector stakeholders as a preliminary step of the national digital health strategy development.
\end{abstract}

Methods: A consultative process was carried out for stakeholders at the national and subnational level to capture high level digital health needs of the government health sector in Sri Lanka. Stakeholders were gathered for a consultative workshop and divided according to their institutional level and influential capacity. Stakeholder needs were captured through structured discussions. The needs were analysed according to the eHealth building blocks of WHO National eHealth strategy toolkit.

Results: Majority of the national level administrator and policy maker needs were the processes and solutions addressing legislation, policy and compliance (40\%). Requirements of PDHS/RDHS were primarily concentrated on digital health services and applications (69\%) which are essential for their institutions. Hospital sector was predominantly concerned about services and applications (71\%). Programmes and campaigns were concerned mainly about the overarching digital health standards and interoperability (33\%) and service-related applications (33\%). Professional bodies required systems and applications on human resource management (53\%).

Conclusions: The digital health needs of the policy makers were more focused in the direction of legislation, policy and compliance while provincial, preventive and curative institutions were focusing on services and applications. Even though there are multiple vertical solutions running independently, suboptimal interoperability between the systems was highlighted. Hence, there is a necessity of a national digital health strategy to align services, solutions, guidelines and standards addressing the national to sub-national digital health needs.

Keywords: Digital health needs, Need assessment, Digital health Sri Lanka, Digital health strategy 